\title{
Selecting and Setting of Protection Appliance for Factory Low- Voltage Power Distribution
}

\author{
Xiaoyan HUANG \\ Sichuan College of Architectural Technology, Deyang, Sichuan, 618000,China \\ email: hxy681018@163.com
}

Keywords: Protection appliance; Setting current; Short circuit protection; Selective type breaker ; Over-current release.

Abstract. This paper discusses the features and applications of the low voltage circuit breakers and fuses, the correct selection and setting of protection appliance for plant power distribution system are analyzed, effectively avoid of the accident and the loss caused by improper selection and setting of low-voltage protective appliance in practical application. The paper has a strong practical value for the power distribution design personnel、operation and maintenance personnel.

\section{Introduction}

Protection appliance occupies an important position for power distribution system at the plant. It may cause serious consequences for the industrial production, if power supply is suddenly interrupted, such as major equipment damage, a large number of discarded products etc, therefore. It is mainly low voltage fuse and breaker cutting off the fault circuit and mainly used for breaker for plant power supply system, if it is not correct for selection and setting of protection appliance in design, it will can't cut off the fault circuit within the required time. If the setting in design is not correct, thus it will damage the wire cable or even expand the scope of power failure. It is especially important to correct selection and set the protective appliance.

\section{The Provision of Power Distribution Lines Protection}

Distribution lines should be installed for short circuit protection, overload protection and grounding fault protection on the basis of "low voltage power distribution design specification" (GB50054 - 2011) in order to guarantee personal safety, reliable electricity and prevent major damage caused by circuit fault. Distribution line protection is to prevent the two aspects of the accident : one is to prevent electric shock caused by indirect contact , the other is to prevent damage caused by overheating for the circuit fault and even lead to fire.

\section{The Selection of Protection Appliance}

\subsection{Selection suggestion of breakers and fuses}

There are the following several points in the selection :

(1) It should be used for circuit breaker as remote control, automatic conversion and residual current protection; (2)It is suggested to be used for selection type breaker with short time delay release in the low voltage distribution panel leads to the feeder, as circuit calculation current is large(such as 400 500A and above); (3) It is suggested

to be used for fuse in distribution line intermediate levels. (4)It should be used for non selection type circuit breaker connected to the motor in end loop; (5)It should be used for miniature circuit breaker in the end of the loop connected with lighting and small appliances far away from the substation.

3.2 The selection principle of the protection characteristic for protection appliance

Setting parameters of protection appliance should ensure that electrical equipment (such as 
motor、 lighting etc. ) starting not to cut off the circuit , in order to ensure the normal starting and running; protection appliance must be cut off the fault circuit within the given time , it is the basic task of protection apparatus ; all levels of protection of electrical distribution system requires a selective action, namely when a failure occurs, it should make the protection appliance cutting off near the fault point cut, and the superior level and a few level ( by the power supply side direction is up ) protection appliance not action, make the power cut scope to the minimum.

\subsection{A example of protection appliance selection for plant power distribution system}

The following illustrate selection of protection appliance in the different position, as shown in Fig.1

Protective appliance for plant power distribution system, should not only to meet reliable action as line is failure, should also meet the selective action , namely, when a fault occurs, should make the protective appliance near the fault point is cut off, and the upper level and a few level (on the power supply side direction is up) protective appliance not action, in order to ensure minimum outage range.In Fig.1 , if the D bit short circuit, should make the RD4 disconnection, the C bit short circuit, should make the RD3 disconnection. If the selectivity is difficult to obtain the guarantee, should protect appliances enable low main trunk (QF2 in Fig.1) will not override disconnected, selective would rather sacrifice the lower distribution line protection (such as D point short-circuit RD3 leapfrog disconnected), the scope of the impact is relatively small.

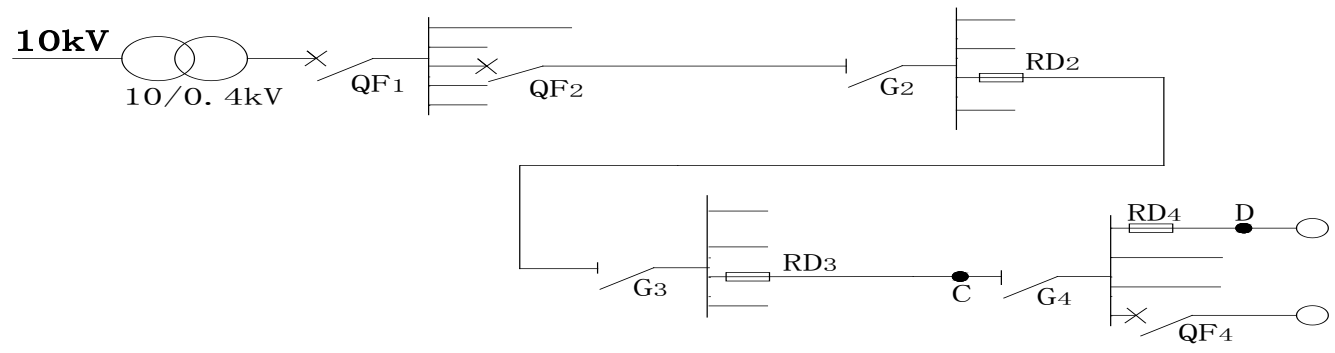

Fig.1. A diagram of the plant low-voltage distribution system

(1)The first protection appliances of distribution lines (namely protection appliance for low main switch cabinet) (QF2 in Fig.1)

Low voltage main switch cabinet protection appliances should put the reliability of power supply in the primary position, to ensure continuous power supply, due to the low pressure protection appliances close to the power transformer, main trunk line has larger capacity, in order to ensure the reliable cut off fault in the first end of trunk line, it should choose selective type circuit breaker with short time delay tripping device.

\section{( 2 ) Second level protection appliance for distribution lines (RD2 in Fig.1)}

General should used fuse of inverse time protection characteristic. When this section of trunk line power supply range is larger, load current calculation is large (above 400A), it can be used selective circuit breaker

( 3 ) The end of circuit (namely terminal distribution box protection appliance)

Protection appliance is directly connected to the electrical equipment (QF4 in Fig.1), it should be installed with a short circuit and ground fault protection, there is no selectivity requirement, it usually use non selection type circuit breaker or leakage circuit breaker.

( 4 ) The upper level protective appliance on the terminal circuit (RD3 in Fig.1)

To ensure the selective action, intermediate levels of multi-stage distribution line should use fuses, according to $1.6: 1$ to pick.

( 5 ) For the important equipment, at all levels should use intelligent circuit breaker to ensure selective coordination.

In summary, selection scheme of protective appliance for plant power distribution line is more 
reasonable for :

Selective type breaker (head end) $\rightarrow$ fuse $\rightarrow$ fuse $\rightarrow$ non selective type breaker or leakage breaker (end).

\subsection{The existing main problems and the solutions of protection appliance setting and selecting}

(1) The existing problems for the total switch selection in LV side of transformer

As shown QF1 in Fig. 1, the breaker should be installed with isolation function. Because line distance between the total switch in low voltage side of transformer and the inferior protection appliance is very close, the probability is very small occurs short circuit and ground fault in this range, if setting the instantaneous over-current protection and this level of protection appliance choice is not good, it may lead to the expansion of the scope of power failure.

(2) Setting and selection of distribution box incoming switch ( G3 and G4 in Fig. 1 )

Because the distribution box each loop outlet are installed protective appliance, then it will increased protection series if installed protective appliances, it should be installed isolating switch.

(3) Application of grounding protection circuit breaker

Grounding protection breaker is first considered for substation low voltage distribution panel leads to the distribution main trunk line, especially larger capacity (above 400A) of the distribution main line at plant, breaker instantaneous over-current release is often difficult to meet the grounding fault protection sensitivity, the feeder can be total grasped installed with a grounding protection breaker.

(4) Line intermediate to replace the fuse with miniature circuit breaker

Due to miniature circuit breaker, generally is non selective circuit breaker, when line fault occurs, it can't cut off the fault selectively. Miniature circuit breaker is only suitable for the terminal loop, it can't be used for line intermediate, so can't replace the fuse freely.

(5) End of line to replace leakage breaker with the ordinary breaker

When a failure occurs, the leakage breaker is not timely breaking, mainly wiring error; or replace leakage breaker with the ordinary breaker.

People can easily come into contact with exposed conductive parts in end of line, the possibility of electric shock is bigger, especially supply portable or mobile electrical equipment is even more so, it usually should use leakage protector at the end of the line.

\section{Inter Stage Coordination Technology Between the Superior Level and the Inferior Protection Appliances in the Factory Power Distribution System}

\subsection{Inter stage coordination characteristics of low voltage protection appliances}

The so-called inter stage coordination, refers to the selective action. Low voltage circuit breaker are divided into selection type and non selection breaker two categories, the former is only with inverse time and instantaneous over-current release; the latter besides short time delay over-current release. So it is a mark of the selection action with short delay. Inverse time release is used as overload protection, the instantaneous release is used as a short circuit and ground fault protection, the following specific analysis on selective for protection appliances between the superior level and the inferior.

\subsection{The superior level use fuse, the inferior use non selective type breaker}

This way requires that time - current curve of the fuse is above the circuit breaker protection curve, and requires that crossing point current Ix is greater than maximum short-circuit current Ik the circuit breaker may through in order to ensure the selective action, as shown in fig. 2. 


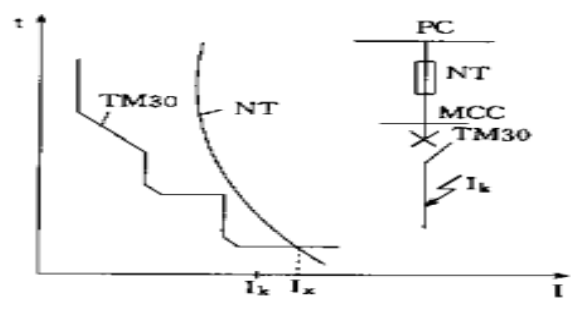

Fig.2. A coordination diagram between the fuse and non selective circuit breaker 4.3 The superior level use selective type breaker, the inferior use fuse

The superior select circuit breaker with short time delay function, it request that the fuse time-current curve is under circuit breaker protection curve, As long as select rated current value of fuse is much lower than the circuit breaker, and I set2 value of the superior level breaker in the premise of satisfying the formula $I d \geq 1.3$ I set2 should be setting bigger, can meet the requirement of selective, as shown in figure 3 .

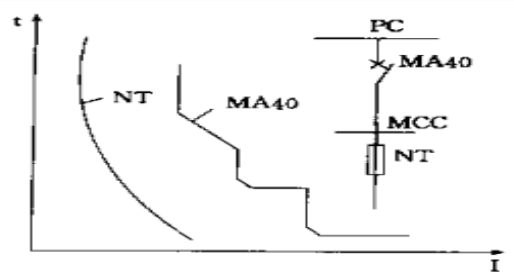

Fig. 3. A coordination diagram between selective circuit breaker and fuses

\subsection{The superior level and the inferior both use non selective circuit breaker}

Long delay time setting value I set1 of superior level circuit breaker A and inferior circuit breaker $\mathrm{B}$, and the instantaneous setting value I set3 are shown in figure 4 . When breaker B later any point (for example, E point) is failure , If the fault current Id $<1500 \mathrm{~A}$, circuit breaker A and B can't instantaneous action, it don't meet the requirements of protection sensitivity ; When $1500 \mathrm{~A}<\mathrm{I}$ d $<3000$ A, B operate, A doesn't operate, it is selective; when $I_{d}>3000 \mathrm{~A}, \mathrm{~A}$ and B are operated, it isn't selective. Therefore, this way is not recommended to use.

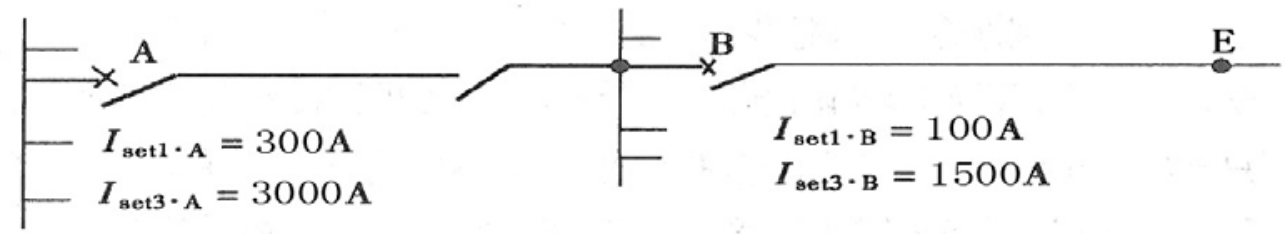

Fig. 4. A diagram of non selective type breaker for both the superior and the inferior

4.5 The superior level use selective type circuit breaker, the inferior use non selective type breaker

This should be coordinated with good selectivity, but must be properly tuning all parameters Figure 5 example, if the setting value of long delay of inferior breaker B I set1.B $=400 \mathrm{~A}$, instantaneous setting value I set3.B=3000 A; I set1.A of superior level breaker A is usually much larger than that of I set1.B , hypothesis I set1.A = 1100 A,

I set2.A setting value should be meet the requirement I set2.A $\geq 1.2$ I set3.B ;

I set3.A should meet under the premise of sensitivity, try setting bigger. 


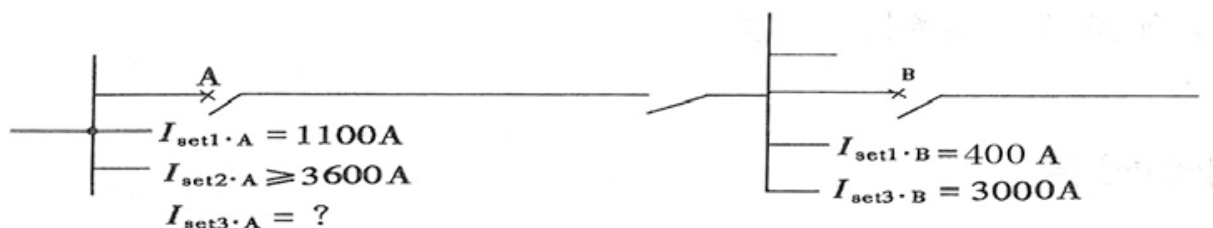

Fig.5. A protection diagram selective type and non selective type breaker

\section{A Example of Setting Value Selection for Factory LV Protection Appliance}

A factory, transformer capacity is $1000 \mathrm{kVA}, 10 / 0.4 \mathrm{kV}$, connection mode is

$\mathrm{Y}$,yn0 ,short circuit capacity for $10 \mathrm{kV}$ side is $200 \mathrm{MVA}$, the main trunk line length leading from the low pressure screen is $200 \mathrm{~m}$, line length from the transformer to the main breaker is $12 \mathrm{~m}$, trunk calculation current IC $=1020 \mathrm{~A}$, ground mode is TN-S, the branch of trunk line connect 7 distribution box , the biggest fuse $\mathrm{Ir}=315 \mathrm{~A}$, the biggest breaker $\mathrm{I}$ set1 $=200 \mathrm{~A}, \quad I$ set3 $=3000 \mathrm{~A}$, as shown in Figure 6.

Requirement : setting parameter of the circuit breaker for the main trunk line

Selection notes : Representative selection two points (the head and the end points ) of the main breaker ,the three-phase short circuit current IK3 calculated values and the grounding fault current value Id1 ,marked in Figure 6.

(1). Determine of setting current I set1 for the long time-delay over-current release

In the environment temperature of $35^{\circ} \mathrm{C}$, the conductor allows continuous load

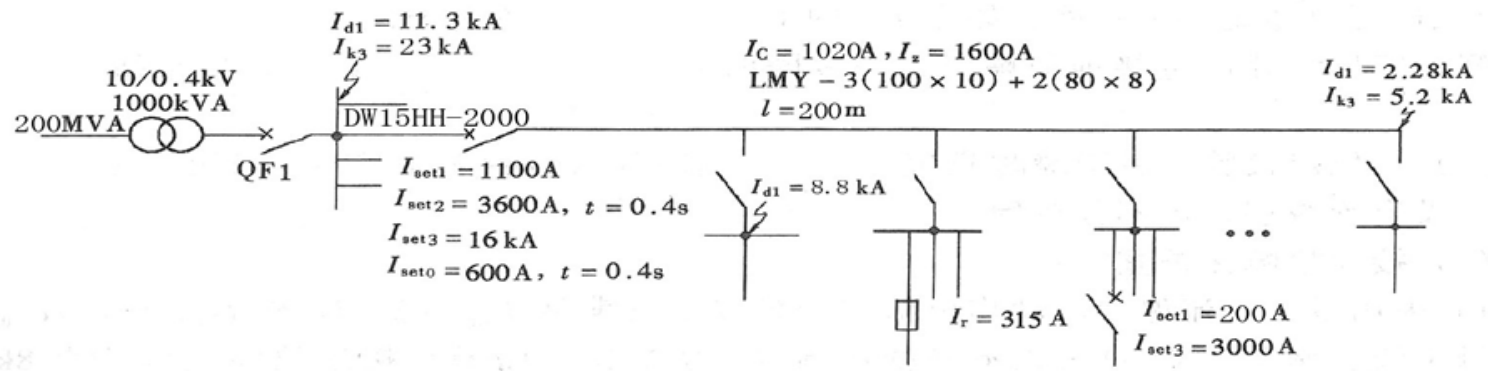

Fig. 6 A diagram of power distribution system

flow IZ = 1600A , according to the overload protection requirements, should comply with IC $\leq$ I set $1 \leq \mathrm{IZ}$, namely I set 1 should be greater than $1020 \mathrm{~A}$ and less than $1600 \mathrm{~A}$, can select $\mathrm{I}$ set $1=1100$ A.

(2). Determine of setting current I set2 for the short time-delay over-current release

According to the "Handbook of industrial and civil power distribution design" (third edition) I set2A setting value should meet the requirement under the type:

$$
\mathrm{I}_{\text {set } 2 \mathrm{~A}} \geq 1.2 \mathrm{I}_{\text {set } 3 \mathrm{~B}}=1.2 \times 3000=3600 \mathrm{~A}
$$

And according to the "low voltage power distribution design specification" (GB 50054-2011), short circuit current protected line on the end should not be less than 1.3 times I set2, namely IK3 $\geq 1.3$ I set2,IK3=5200A(as shown in Fig.6) , therefore

$$
\mathrm{I}_{\text {set2 }} \leq \frac{I_{K 3}}{1.3}=\frac{5200}{1.3}=4000 \mathrm{~A}
$$


Comprehensive (1), ( 2) two formula, can select I set2 =3600A

(3). Determine of setting current I set3 for the instantaneous over-current release

According to the operation experience, I set3 in satisfying short circuit action conditions should try setting bigger, select I set $3=15 \mathrm{I}$ set $1=15 \times 1100 \mathrm{~A}=16.5 \mathrm{k} A$, therefore, when recently a distribution box bus generated at the grounding fault, will not cause the instantaneous tripping.

\section{References}

[1] Design specification of low voltage distribution( GB 50054-2011), [M] .Beijing: China Planning Press, 2012.

[2] Yuanhui Ren and so on, Handbook of industrial and civil power distribution design (Third Edition ), [M]. Beijing: China electric power press, 2005.

[3] General electrical equipment distribution design specification (GB50055-2011) , [M].Beijing: China Planning Press. 2012

[4] Yuejun Xie, New type low voltage circuit breaker and low-voltage protection equipment level coordination, [J]. Rural electrification, 2002.

[5] Fan Yang, low voltage protection apparatus: the chest has the global, reasonable selection,[J].Building Electrical applications, 2010(15).

[6] Yuanhui Ren, The development of protection for LV distribution line - The application of grounding protection circuit breaker,[J].Low voltage electrical apparatus, 1991(11-12). 